\title{
The Viability of Spectrum Trading Markets
}

\author{
Carlos E. Caicedo, Syracuse University
}

Martin B. H. Weiss, University of Pittsburgh

\begin{abstract}
Spectrum trading markets are of growing interest to many spectrum management agencies. They are motivated by their desire to increase the use of market-based mechanisms for spectrum management and reduce their emphasis on command and control methods. Despite the liberalization of regulations on spectrum trading in some countries, spectrum markets have not yet emerged as a key spectrum assignment component. The lack of liquidity in these markets is sometimes cited as a primary factor in this outcome. This work focuses on determining the conditions for viability of spectrum trading markets. We make use of agentbased computational economics to analyze different market scenario and the behaviors of its participants. Our results provide guidelines regarding the number of market participants and the amount of tradable spectrum that should be present in a spectrum trading market for it to be viable. We use the results of this analysis to make recommendations for the design of these markets.
\end{abstract}

\section{INTRODUCTION}

The radio frequency spectrum is a highly regulated resource whose management is usually deferred to a government agency in most countries. The tasks related to spectrum management encompass all the activities related to regulating this resource, including spectrum allocation and assignment of spectrum as well as regulation enforcement activities. For our purposes, spectrum allocation refers to defining acceptable uses of certain bands (e.g., FM radio), whereas spectrum assignment is the process of granting rights to particular users in a band that has been allocated (e.g., a radio station).

Traditional spectrum allocation and assignment mechanisms have focused on avoiding interference between users and on the type of use given to spectrum rather than on the efficient use of spectrum and the maximization of economic benefits. Due to this, most of the spectrum is used suboptimally most of the time with low average occupancy values (less than 10 percent as reported in [1]).

Additionally, managing spectrum has become increasingly difficult for regulatory agencies due to the new technologies and uses for spectrum that are continuously emerging and placing increasing demands on this resource. Thus, more flexible assignment mechanisms have to be put in place to adjust to this new reality while still achieving the best usage of spectrum possible under economic or social welfare considerations [2].

Spectrum auctions have become a common technique for regulatory agencies to assign spectrum to new users. Once the auction is over, however, the license holders do not get feedback about the current valuation of spectrum. For economically driven spectrum assignment to be optimally effective, a secondary market must exist that allows spectrum users to optimally choose between capital investment and spectrum use on a continuous basis, not just at the time of initial assignment [2]. The interactions in the market should take into account the geographic reusability and non-perishable characteristics of spectrum, which make its trading different than trading traditional market commodities.

Unlike much of the dynamic spectrum assignment (DSA) literature, which focuses on noncooperative sharing, spectrum trading is a form of primary cooperative spectrum sharing that involves permanent license transfers for economic consideration [3]. Thus, it assigns spectrum to those who value it most, allowing for the establishment of dynamic market-driven and competitive wireless communication markets.

Spectrum trading markets are of growing interest to many spectrum management agencies. They are motivated by their desire to increase the use of market-based mechanisms for spectrum management to increase spectrum efficiency. The research reported here is, in many ways, a best case analysis to determine the viability of those markets.

\section{PARTICIPANTS IN A SPECTRUM TRAdING MARKET}

To understand the organization of and interactions in a spectrum trading (ST) market, we need to know what entities participate in such a market. In [4] we elaborated a classification for market structures that support ST. This classification considered two main types of market 
structures for spectrum trading: over-the-counter (OTC) markets and exchange-based market operation. We focus on the exchange-based case in this article.

Figure 1 illustrates an exchange-based trading scenario. In this scenario the exchange collects the offers to sell and offers to buy (bids) spectrum, determines the winning bid, and transfers the spectrum usage right from the selling spectrum user to the new owner of that right. The entities that participate in exchange-based ST markets are the following.

\section{SPECTRUM EXCHANGE}

An entity that provides and maintains a marketplace or facilities for bringing together buyers and sellers of spectrum in which spectrum trading transactions can take place. It also publicizes prices and anonymizes trading entities.

\section{SPECTRUM USER}

An entity that uses spectrum for a particular purpose. A spectrum user (SU) might be acting in one of the following roles at a given moment in time:

- Spectrum license holder (SLH): An entity that owns a spectrum license and offers it for trading in exchange for financial compensation. This entity can be a wireless service provider, market maker, or spectrum exchange that has been assigned a spectrum trading band by a regulatory agency. In general, SLHs hold spectrum for speculation or for their own use.

- Spectrum license requestor (SLR): An entity that submits bids for spectrum licenses to the ST market with the intent to acquire the license. SLRs obtain spectrum for speculation or their own use. An entity that acts as an SLR can be a wireless service provider, market maker, or company/enterprise that acquires spectrum on behalf of another.

\section{SPECTRUM REgULATOR}

A spectrum regulator is a government entity that oversees the ST market and defines the regulations for its operation. It is also responsible for maintaining a spectrum availability and assignment database which is updated every time a spectrum trade is completed to register the identity of the new holder of spectrum.

\section{MARKet MAKers}

A market maker facilitates trading; it does not provide services with its inventory. It acts as a dealer that holds an inventory of spectrum and stands ready to trade when an SLR (buyer) or SLH (seller) desires. It gets revenue through the spread between the sell and buy prices for spectrum, and holds a spectrum inventory for negotiating and speculating.

\section{EXCHANGE-BASED ST MARKETS}

In exchange-based ST markets, the spectrum exchange is the central entity for market operation. In general, an exchange denotes the idea of a central facility where buyers and sellers can transact and which may charge fees for its ser-

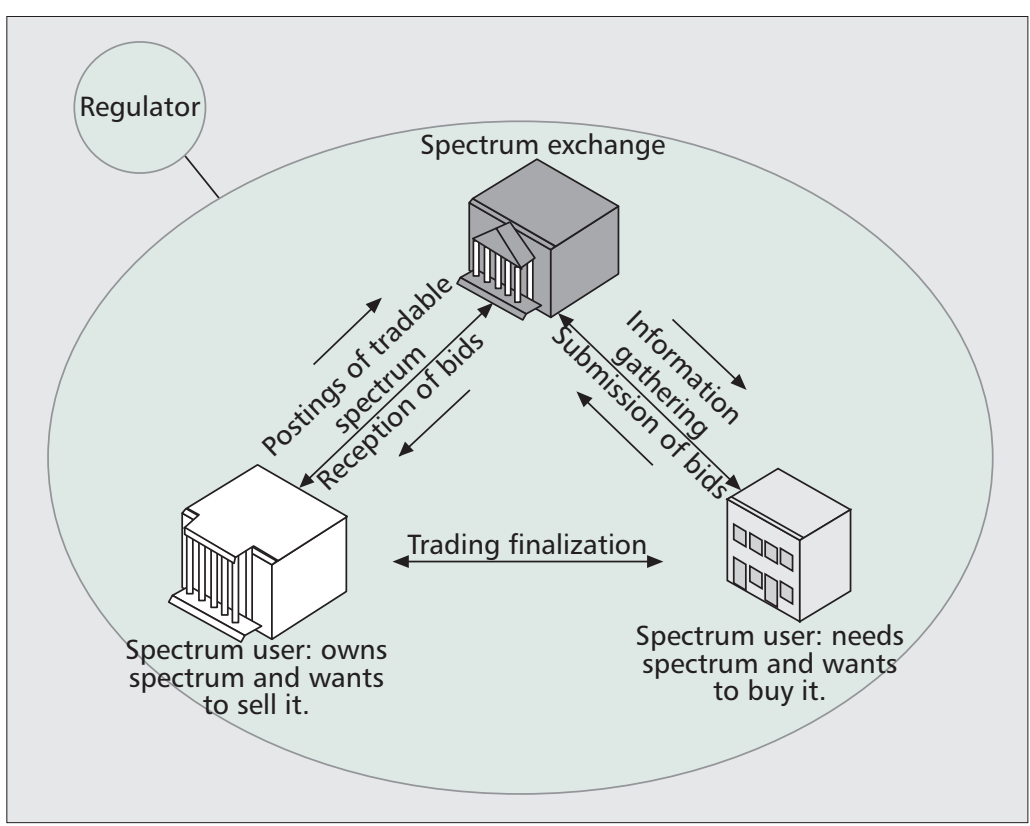

Figure 1. Spectrum trading scenario.

vices. In the traditional sense, an exchange is usually involved in the delivery of the product. For a spectrum exchange to allow use of traded spectrum, the required devices do not need to be collocated in the exchange, so the exchange might not be involved in the delivery of service. We assume that spectrum exchanges make use of continuous double auctions as a mechanism to match buyers and sellers.

We consider that the spectrum exchange acts as a pooling point (POOL) if its facilities house the communication equipment that enable the delivery of wireless services through spectrum acquired by a buyer in the exchange. This kind of exchange also takes care of the configuration of equipment required to make the spectrum usable to the new license holder. A non-pooling point exchange (NOPOOL) only delivers the authorization for use of spectrum to the buying party in a spectrum trade. The new SLH must then use this authorization to configure its devices to make use of the spectrum it has just acquired.

From a functional perspective a spectrum exchange can be a band manager (BM) for a given segment of spectrum over a region or have no band manager functionality (NOBM). BM exchanges support-leasing arrangements in addition to permanent license transfers. In contrast to BM exchanges, a NOBM exchange will only facilitate the trading of spectrum among entities in the market without holding any spectrum inventory itself.

For scenarios where the exchange has BM functionality, SLRs send a request for spectrum to the exchange, which, if possible, will assign spectrum to the requesting entity in the form of a timed lease within the band managed by the exchange. For a NOBM exchange the spectrum it will handle for trading will come from market participants that use the exchange and make bids and offers of spectrum. It is worth mentioning that unless the market has defined a basic 


\begin{tabular}{|c|c|}
\hline Exchange type & Characteristics \\
\hline POOL_BM & $\begin{array}{l}\text { Pooling point }+ \text { band manager functionality } \\
\text { - Use of traded spectrum is enabled and configured through equipment/infrastructure owned by the exchange. } \\
\text { - All tradable spectrum is held by the exchange } \\
\text { - All tradable spectrum returns to or is given by the exchange }\end{array}$ \\
\hline POOL_NOBM & $\begin{array}{l}\text { Pooling point only, no band manager functionality } \\
\text { - Use of traded spectrum is enabled and configured through equipment/infrastructure owned by the exchange. } \\
\text { - Different segments of spectrum can be activated and configured through the equipment/infrastructure of the } \\
\text { exchange } \\
\text { - No spectrum inventory is held by the exchange }\end{array}$ \\
\hline NOPOOL_BM & $\begin{array}{l}\text { Non-pooling point }+ \text { band manager functionality } \\
\text { - All tradable spectrum is held by the exchange } \\
\text { - All tradable spectrum returns to or is given by the exchange } \\
\text { - Exchange grants authorizations for use of spectrum (no equipment configuration is done by the exchange) }\end{array}$ \\
\hline NOPOOL_NOBM & $\begin{array}{l}\text { Non-pooling point, no band manager functionality } \\
\text { - No spectrum inventory is held by the exchange } \\
\text { - Exchange grants authorizations for use of spectrum (no equipment configuration is done by the exchange) }\end{array}$ \\
\hline
\end{tabular}

Table 1. Types of exchanges.

amount of bandwidth as a spectrum-trading unit, it will be very complicated to match bids and offers of spectrum without incurring wasteful assignment of this resource. Although giving a particular structure to the way a spectrum-trading band should be segmented will limit its operational flexibility, it also provides benefits in terms of simplifying the specifications to characterize a particular spectrum trade and managing interference between ST users.

From the previous discussion, the proposed classification generates four types of spectrum exchanges that can be used to implement an ST market. These are listed in Table 1.

\section{MODELING OF ST MARKETS}

We use agent-based computational economics (ACE) to analyze spectrum trading markets and the behaviors of their participants which, given their variety, would be difficult to study with conventional statistical and analytical tools. ACE is "the computational study of economic processes modeled as dynamic systems of interacting agents" [5]. An agent in an ACE model is a software entity with defined data and behavior. Agents can represent individuals, institutions, firms, and physical entities. ACE methods have been used to study cooperative secondary spectrum sharing (in which temporary usage rights are negotiated) [6].

A spectrum trading market modeling tool (SPECTRAD) has been developed as part of our research work, and makes use of ACE methods and concepts. With this tool, we model the participants in a ST market over a set of different scenarios. Our focus is to determine the conditions for viability of these markets. We define a viable spectrum trading market as one that possesses good liquidity and sustainability characteristics. As a first step in our analysis we have chosen to examine spectrum markets where only one wireless standard is used, and the unit segments of tradable frequency have equal operational conditions (fungibility). Future work will examine more complicated and realistic scenarios. This is the best case scenario for liquidity.

When modeling markets, the agents representing market participants have limited (if any) knowledge of the decisions and state of other market participants (bounded rationality). Agents adapt their behavior based on their goals, and their interaction with the market and/or other agents. In ACE modeling, once initial conditions have been specified, the evolution of the model is only dependent on the interactions among agents, and given the diversity of interactions that can arise, it is difficult to perform straightforward causal analysis by tracking one market participant. Thus, ACE models provide a tool to observe the aggregate behaviors that emerge on a system from the individual behaviors of its components (agents). Analysis of these behaviors over several scenarios can provide insights into characteristics of new markets, the effect of economic policies, and the roles of institutions.

By characterizing the trading, information overhead, and infrastructure costs of different ST market implementation architectures, and since we are interested in the running behavior (sustainability) of the market once its operating infrastructure has been put in place, we find that the only differentiating factor between them is whether the exchange is organized to work as a band manager (BM) or not (NOBM) [2]. For the sake of brevity we mention our model results with NOBM scenarios in this article.

The following subsections describe the assumptions and behaviors of the market entities used in our models. Further details of the implementation of our models can be found in [2].

\section{General Market Setup and MOdEL AsSUMPTIONS}

The following are the assumptions used in our models:

- Interference conditions do not impact the services provided over a unit of traded spectrum. 
- Trading takes place over an exchange entity and over a single geographic service area in which the wireless services providers (modeled by SU agents) have enough radio base stations to provide service coverage.

A market scenario is defined by the following set of parameters:

- Number of market participants (numSU)

- Distribution of spectrum users'valuation level $(L)$

- Amount of available spectrum for trading $(S)$

In the market scenarios considered, wireless service requests manifest to each $\mathrm{SU}$ as requests of traffic to be served for which the SU has to determine if it has sufficient resources. The SUs can obtain resources to serve traffic by either acquiring spectrum in the form of basic bandwidth units (BBUs) or using a unit of transmission of an alternate technology (AT).

Investment in AT transmission units can resemble investing in equipment to make better use of spectrum already owned by the SU or in wireline technology, thus avoiding the purchase of additional BBUs. The choice between BBUs or ATs will be based on the economic benefit a given SU might receive from making a selection as it tries to minimize its costs for providing wireless service. Each SU will have a fixed price for its choice of AT unit which does not change during the life of the market. Thus, if an SU is acting as a spectrum license requestor (i.e., buyer) when the market price for a BBU is higher than its AT price, the SU will buy ATs; and when BBU prices are lower or equal to its AT price, the SU will buy BBUs.

\section{BehaVIORS For ST MARKet ENTITIES}

Spectrum User Behavior - Spectrum users are the agents that model wireless service providers (WSPs), and buy and sell spectrum in order to meet traffic requests (buy) or obtain economic gain (sell). For our analysis we model the aggregate traffic demand for each SU within the ST service area with an exponential distribution with a mean of $\mu_{\text {traffic }}$. The interval between changes of traffic demand is modeled as an exponential distribution with a mean of $\mu_{\text {tchange }}$. In this model we assume that the traffic demands faced by SUs are not correlated.

The SUs submit requests to buy (bids) or sell (asks) to the exchange. The exchange collects these requests and tries to find the best match between requests to establish a trade. The SU can query the exchange for its current market quote, which contains the minimum ask and maximum bid price posted in the market. SUs use this information in their pricing decisions. Additionally, an SU can post buy/sell orders that should execute immediately at the current best price of the market (market order) or specify to the exchange its desire to buy/sell BBUs at the best price possible but in no event pay/sell for more/less than a specified limit price (limit orders). If an SU buys AT units, it is aware that they have a finite lifetime and should be decommissioned in the future based on their mean lifetime.

NOBM Spectrum Exchange Behavior - We assume a continuous order-driven market in which SUs may trade at any time they choose.
After each order is posted, the exchange updates its order book, and if a trade can take place, it transfers the spectrum license from the seller to the buyer and records the details of the trading transaction. It also informs the regulator agent about the trade so that it can keep track of the owner of each BBU.

After each trade or if there was no trade, the exchange announces the market quote, informing market participants of the current market ask price (best price at which spectrum is being sold in the market) and the current market bid price (price of the best offer to buy spectrum in the market). This allows market participants to adapt their price behavior to make competitive bids or asks in the future.

Market Maker - The market maker (MM) provides liquidity to the market and corrects market imbalances. In our model the MM agent stands ready to make bids for spectrum if no SU is posting a bid, and it posts an offer to sell if no SU is on the selling side of the market. This makes the MM a very reactive entity that only intervenes in the market when there is a severe imbalance (i.e., no buyers or no sellers) in order to keep the market alive. Using a simplified MM allows us to determine which market scenarios are viable without much economic intervention from entities that do not provide wireless services.

The MM has an initial inventory of BBUs assigned to it which it uses to keep a bid-ask (buy-sell) spread present at all times in the market. When market intervention by the MM is not required, the MM will issue a bid or ask with the objective of getting its spectrum inventory back to its reference level, which is the same as its initial spectrum inventory amount.

Regulator Agent - A regulator agent models a regulator entity, oversees the trades being conducted in the market, and updates a spectrum assignment database so that ownership of a given BBU can be verified if needed.

\section{FACTORS FOR ST MARKET VIABILITY}

Our focus is on determining the conditions for viable ST markets with respect to their liquidity and sustainability characteristics, so we selected a set of parameters/measures that capture the main characteristics of these markets.

- Midpoint price for spectrum BBU: This price gives an indication of the average price at which a BBU is being valued in the market. Low values of this measure indicate an excess in supply or low spectrum demand in the market, while high values indicate low supply or high demand for spectrum.

- Relative bid/ask spread: The bid/ask spread is the difference between the best sell price and the best buy price in the market. The relative bid/ask spread is the size of the bid/ask spread relative to the midpoint price of the quoted asset. This factor can be used as an indicator of the liquidity of a market $[7,8]$. In other words, high values of this parameter indicate low liquidity in the market, while low values would indicate high liquidity.
In our model the

market maker agent

stands ready to

make bids for

spectrum if no SU is

posting a bid, and it

posts an offer to sell

if no SU is on the

selling side of the

market. This makes

the market maker a

very reactive entity

that only intervenes

in the market when

there is a severe

imbalance. 


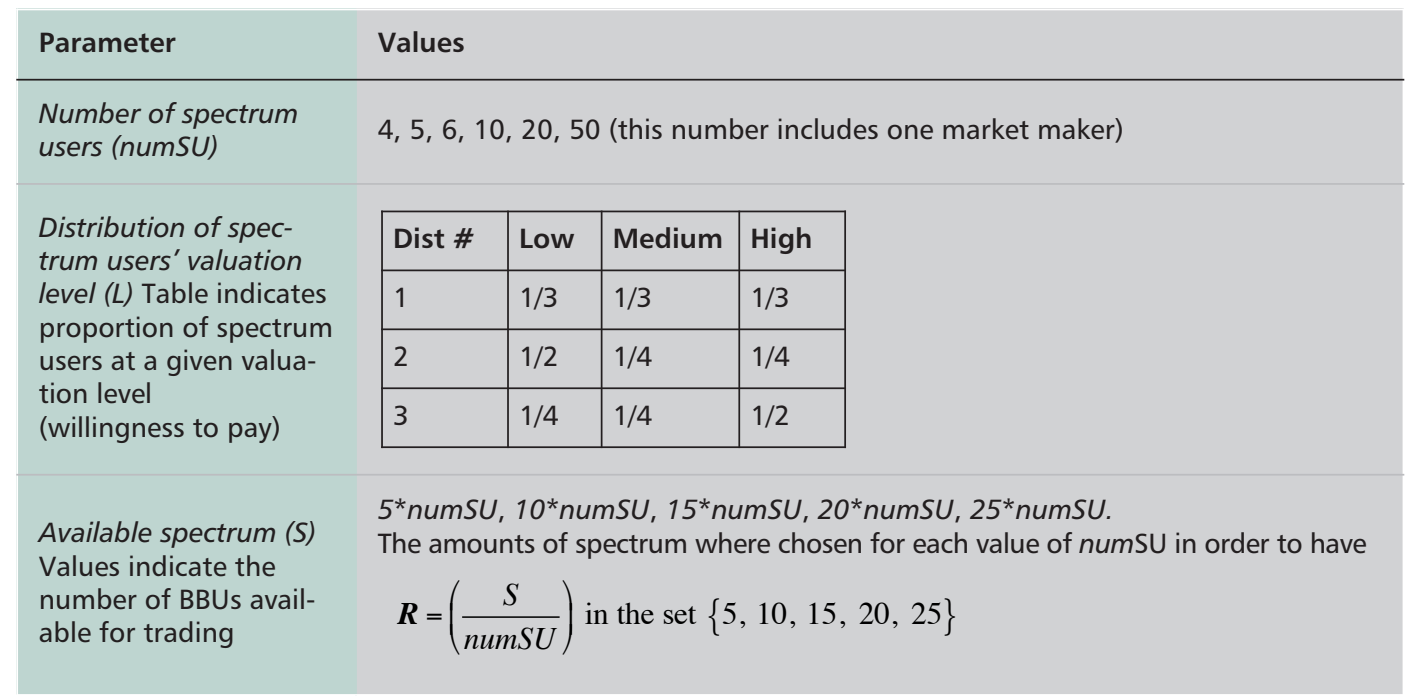

Table 2. Parameters for modeled markets.

\begin{tabular}{|lllll}
\hline ID & Factor & Pass (P) & Fail (F) & Score P/F \\
\hline C1 & Percentage of completed market runs & $\geq 70 \%$ & $\leq 50 \%$ & $2 /-2$ \\
\hline C2 & Relative bid-ask spread & $\leq 20 \%$ & $\geq 50 \%$ & $1 /-1$ \\
\hline C3 & Mid-point BBU price & $\geq 100$ & $\leq 25$ & $1 /-1$ \\
\hline & $\begin{array}{l}\text { Relative difference of the MM's } \\
\text { inventory to its reference level }\end{array}$ & $\leq 25 \%$ & $\geq 100 \%$ & $1 /-1$ \\
\hline
\end{tabular}

Table 3. Criteria for NOBM market scenario evaluation.

- MM's BBU inventory difference: This parameter tracks the difference between the MM's current spectrum holdings (BBU inventory) and its reference level of inventory units. Substantial deviations from the reference level would indicate problems in the buying or selling side of the market.

-Percentage of offered spectrum: Expressing the amount of spectrum being offered for sale as a percentage of the total spectrum available in the market, we find that if this percentage is high, the majority of the tradable spectrum is not in use by the SUs and thus is being offered for sale. This would indicate low spectrum efficiency. In general, the lower the value of this percentage, the more efficiency there is in terms of spectrum use.

- Number of complete market runs: For the collection of statistics to analyze each market scenario, 100 runs of each scenario are performed in SPECTRAD. Activity in a market starts with a series of mock auctions so that the SUs can find an initial starting price for trading. If this initial phase is not successful in finding a starting price, the market does not proceed to actual spectrum trading. This factor counts how many of the attempted market runs were successful in finding a stable starting price and thus able to initiate spectrum trading. It is useful as an indicator of market viability since a high percentage of complete market runs indicates that initiating trading is feasible and sustainable with- out difficulty. In contrast, having a low percentage of complete market runs would indicate that the market structure is not well suited to support sustainable trading.

All of these parameters were found to be applicable to NOBM markets. A similar analysis and determination of viability factors was conducted for BM markets, but it is not discussed here. The reader can find more details on BM cases in [9].

\section{ViabiLITY CRITERIA AND ResUlts}

A summary of the values used for the parameters of the market scenarios modeled is shown in Table 2. Each run of the model was executed for 5000 time ticks of which 3000 were used for the warm-up period. Data was collected on the last 2000 time ticks.

In the scenarios modeled, the variation of the tradable spectrum amount and number of SUs are related in such a way that the value of the average number of BBUs per spectrum user $(\boldsymbol{R})$ is in the set $\{5,10,15,20,25\}$. For all scenarios, when $\boldsymbol{R}$ is equal to 10 , on average every spectrum user has enough spectrum to serve its average traffic requirement value. Thus, $\boldsymbol{R}$ values lower than 10 indicate an under-supply of spectrum, while values greater than 10 indicate an over-supply of this resource to attend the average traffic needs of a SU.

In order to determine the viable NOBM markets based on the factors mentioned in the previous section, we developed decision criteria to determine if the behavior of a particular factor in a market is to be considered as desirable/ acceptable or undesirable/unacceptable. Additionally, in order to keep track of the aggregate behavior characteristics of a market, we gave a score to each factor with a positive value when the market complies positively with the desired behavior characteristic or a negative one when it complies with the undesirable behavior criteria. Based on the total scores for a market's behavior, a final list of viable markets was obtained.

Most of the threshold values for each criterion were derived from the simulation data by tak- 
ing into account the values that determine breakpoints for different behaviors for a scenario. Table 3 summarizes the criteria to be used to evaluate and give scores to the different scenarios studied in this work. Factors such as the percentage of completed market runs where given more weight than other factors given their relative importance in the determination of viability characteristics (sustainability in this case).

\section{VIABLE NOBM MARKETS}

Using the list of criteria for scenario evaluation mentioned in Table 3, the scores for the simulated scenarios are summarized in Fig. 2. We consider markets with scores greater than 0 as viable. Scenarios with this condition met several of the desirable conditions for a viable market; in particular, they all have a percentage of running markets greater than 50 percent. We only show the scores for scenarios with user valuation distribution \#1 (as defined in Table 2) since there was no difference in terms of viable markets among scenarios with different valuation level distributions.

Based on the scores, we can say that most of the viable market scenarios are those that have $\boldsymbol{R}$ values that meet the condition $5 \leq \boldsymbol{R} \leq 10$ and a number of spectrum users (numSU) such that $6 \leq n u m S U \leq 50$. When $\boldsymbol{R}=15$, the viable scenarios are those with $10 \leq n u m S U \leq 20$. Figure 3 shows the spectrum efficiency results from our modeled scenarios.

\section{VIABILITY IMPLICATIONS}

NOBM spectrum trading markets are viable under the criteria used in this work and the ideal conditions mentioned earlier for markets with more than 6 spectrum users as long as there is no oversupply of spectrum, that is, when $\boldsymbol{R}=5$ and $\boldsymbol{R}=10$ (although cases with 5 SUs were viable when $\boldsymbol{R}=10$ ).

A value of $\boldsymbol{R}=5$ indicates scenarios where on average there is 50 percent less spectrum per SU to serve the SU's average traffic requirement. A value of $\boldsymbol{R}=10$ is the reference value where the amount of spectrum per user is very close to being enough to serve a SU's average traffic requirement and is where most of the viable scenarios are found. When $\boldsymbol{R}=15$, there is a 50 percent oversupply of spectrum and in this case, the viable markets are those with 10 to 20 spectrum users. Thus, if there is little or no oversupply of spectrum and with a number of spectrum users greater than or equal to 6 , most NOBM spectrum trading markets will be viable.

Some of the implications of the results from the models used in this work are as follows.

Number of Market Participants - Spectrum trading is viable in markets with no excessive oversupply or undersupply of spectrum for a wide range of spectrum user values. However, when the number of SUs is less than 6, NOBM markets are unviable. Regulators and entities interested in these markets must make sure that enough trading participants will be in the market. The results presented here can serve as a guideline, but should be complemented by further study of market environments under different traffic (demand) patterns.

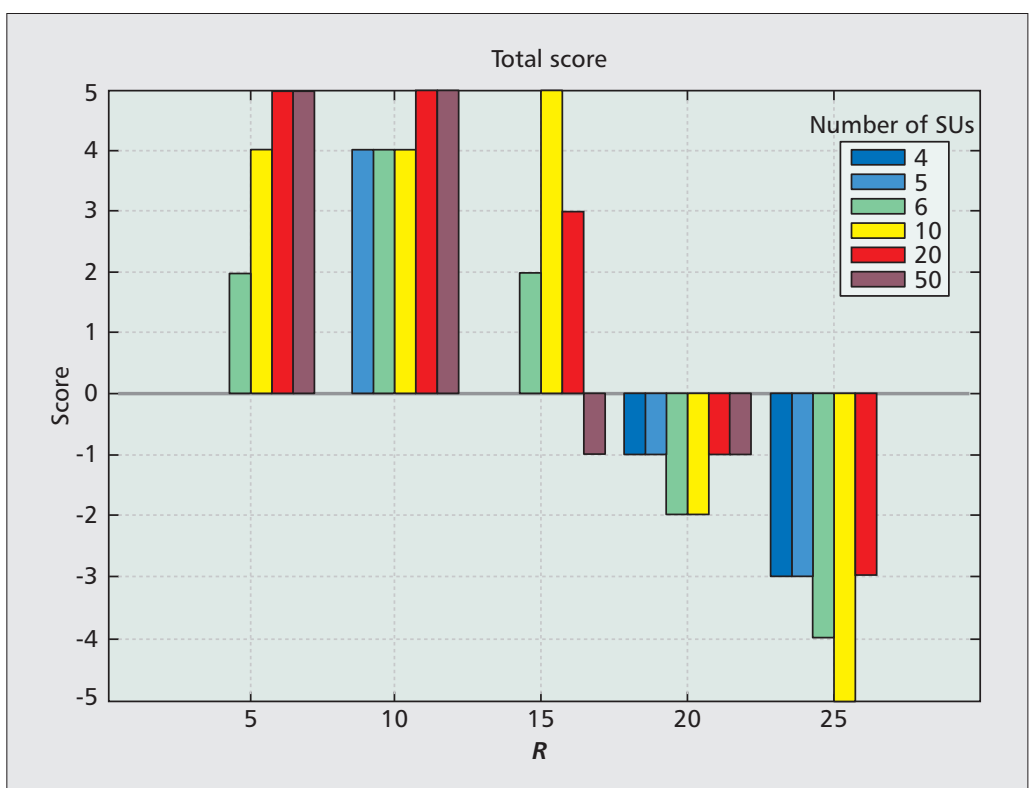

Figure 2. Scores for NOBM scenarios.

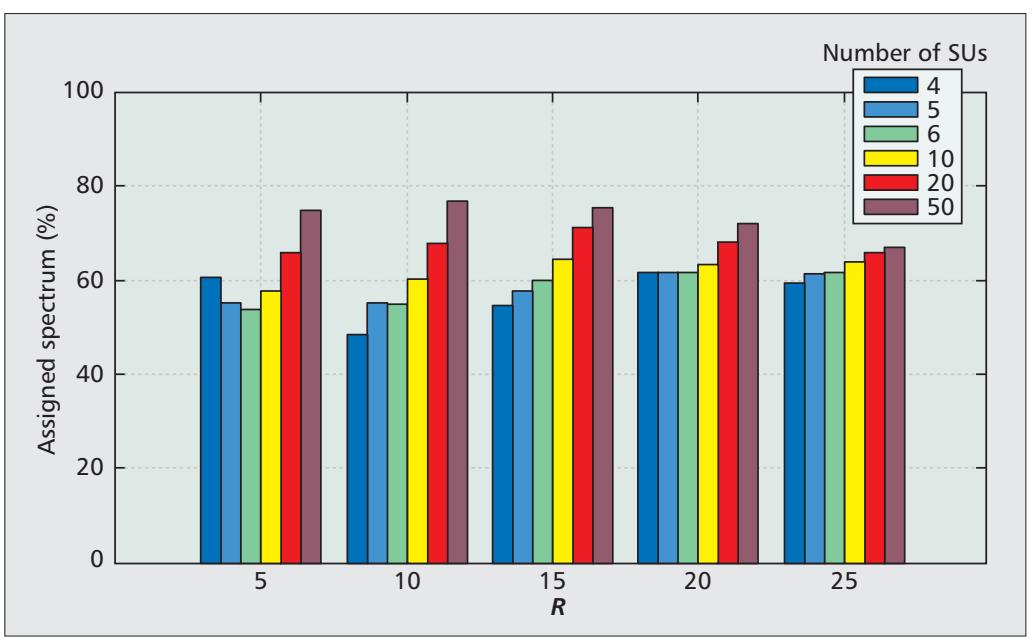

Figure 3. Percentage of assigned spectrum for NOBM scenarios.

Market Makers in a ST Market - Simple MMs as providers of liquidity, like the ones used in the models of this work, help in the establishment of viable markets by holding a spectrum inventory with which they can transact. Thus, regulators will not have to specify complex MM behavior requirements or rules. Since an MM does not make use of its spectrum, assigning too much inventory to an MM would decrease spectrum efficiency. However, the greater the inventory level of the MM, the better prepared it would be to intervene in the market if there is a lack of spectrum offerings. Thus, regulators will need to define the level of spectrum holdings of an MM to reach a desired balance of market viability vs. spectrum efficiency.

\section{Amount of Available Spectrum for Trading}

- Oversupply of spectrum negatively affected all market scenarios considered. In particular, an oversupply of 100 percent above the level of spectrum the SUs need to serve their average 
Our methods and

tools can be extend-

ed to the study of

other telecommuni-

cations markets or

scenarios where

adaptive behaviors of

the system partici-

pants are allowed

and where studying

the emergent behav-

ior of the market is

of interest. traffic needs leads to unviable markets. An adequate amount of spectrum for trading should be made available in the market in order to maintain trading activity. Our scenarios showed viability with spectrum amounts 50 percent above or below the level of spectrum needed to attend average traffic in the market.

Spectrum Efficiency - In the viable ST market scenarios, NOBM markets provided for spectrum efficiencies between 51 and 77 percent under the ideal conditions of this work. These results show a positive characteristic of ST markets that is of great interest to regulators and SUs. Analysis of spectrum efficiency in other market scenarios and conditions is left for future work.

\section{CONCLUSIONS}

The outcomes of our study can help policy makers and wireless service providers (future and current) understand the required conditions for implementing spectrum trading markets. The market and operational scenarios we used assumed ideal conditions among which were the use of a single wireless technology and the fungibility of spectrum in the service area where ST was operating. These restrictions were used to define initial scenarios (almost ideal) over which to study the dynamics of spectrum trading. Future work will make use of our modeling tool (SPECTRAD) to analyze more complicated scenarios.

Our models indicate that spectrum markets can be viable if sufficient numbers of market participants exist and the amount of tradable spectrum is balanced to the demand. Given that a minimum of five to six active spectrum users (wireless service providers) are necessary in a particular service area, it seems unlikely that spectrum markets will be viable in mobile markets unless the barriers to market entry for new service providers are lowered. ST markets may well create the incentive for the appearance of new types of wireless service providers, but without a truly liberalized ST market in place it is unlikely they will do so. Thus, a challenge for regulators and researchers alike will be identifying an appropriate band to promote spectrum trading or to facilitate the entry of new market participants, and perhaps even end users.

The matter of balancing tradable spectrum to demand will prove more challenging for regulators because this requires insight into service demand, even though this need not be too precise. Thus, it will be important to develop useful (and observable) proxies that enable regulators to estimate how well markets are balanced. The viability of spectrum trading in more complicated trading scenarios (i.e., more than one wireless standard and/or non-fungible spectrum) is left for future work.

An important byproduct of our research is demonstrating how ACE can be applied to the study of telecommunication markets. Our methods and tools can be extended to the study of other telecommunications markets or scenarios where adaptive behaviors of the system participants are allowed and studying the emergent behavior of the market is of interest.

\section{REFERENCES}

[1] M. A. McHenry, "NSF Spectrum Occupancy Measurements Project Summary," 2005.

[2] C. E. Caicedo, Technical Architectures and Economic Conditions for the Viability of Spectrum Trading Markets, Ph.D. dissertation, Univ. of Pittsburgh School of Information Sciences, 2009.

[3] M. Weiss and W. Lehr, "Market-Based Approaches for Dynamic Spectrum Assignment," working paper, Nov. 2009; http://d-scholarship.pitt.edu/2824/.

[4] C. E. Caicedo and M. Weiss, "An Analysis of Market Structures and Implementation Architectures for Spectrum Trading Markets," Telecommun. Policy Research Conf., Fairfax, VA, 2008.

[5] L. Tesfatsion, "Agent-Based Computational Economics: A Constructive Approach to Economic Theory," Ch. 16, Handbook of Computational Economics, vol. 2, L. Tesfatsion and K. L. Judd, Eds., North-Holland, 2006, pp. 831-80.

[6] A. Tonmukayakul and M. Weiss, "A Study of Secondary Spectrum Use Using Agent-Based Computational Economics," Netnomics, vol. 9, no. 2, 2008, pp. 125-51.

[7] L. Harris, Trading and Exchanges: Market Microstructure for Practitioners, Oxford Univ. Press, 2003.

[8] T. Chordia, R. Roll, and A. Subrahmanyam, "Liquidity and Market Efficiency," J. Financial Economics, vol. 87, 2008, pp. 249-68.

[9] C. Caicedo and M. Weiss, "The Viability of Spectrum Trading Markets," IEEE DYSPAN '10, Apr. 2010, Singapore.

\section{BIOGRAPHIES}

CARLOS E. CAICEDo BAstidAs (ccaicedo@syr.edu) is an assistant professor and director of the Center for Convergence and Emerging Network Technologies (CCENT) of the School of Information Studies at Syracuse University. He holds a Ph.D. in information science from the University of Pittsburgh, and M.Sc. degrees in electrical engineering from the University of Texas at Austin, and Universidad de los Andes, Colombia. His current research interests are spectrum trading markets, secondary use of spectrum, security, and management of data networks.

MARTIN B. H. Weiss [M'76] holds a Ph.D. in engineering and public policy from Carnegie Mellon University and an M.S.E. in computer, information, and control engineering from the University of Michigan. He is currently a faculty member and associate dean for academic affairs and research at the School of Information Sciences at the University of Pittsburgh. He has performed techno-economic research in telecommunications and telecommunications policy over the past 20 years, and currently works on topics related to cooperative secondary use of electromagnetic spectrum. 